\title{
ARTICLE
}

Received 19 May 2014 | Accepted 2 Sep 2014 | Published 6 Oct $2014 \quad$ DOl: 10.1038/ncomms6141

\section{Unravelling the mechanism of non-ribosomal peptide synthesis by cyclodipeptide synthases}

Mireille Moutiez ${ }^{1, \star}$, Emmanuelle Schmitt ${ }^{2, \star}$, Jérôme Seguin ${ }^{1}$, Robert Thai ${ }^{1}$, Emmanuel Favry ${ }^{1}$ Pascal Belin ${ }^{1}$, Yves Mechulam² \& Muriel Gondry ${ }^{1}$

Cyclodipeptide synthases form cyclodipeptides from two aminoacyl transfer RNAs. They use a ping-pong mechanism that begins with transfer of the aminoacyl moiety of the first aminoacyl tRNA onto a conserved serine, yielding an aminoacyl enzyme. Combining $\mathrm{X}$-ray crystallography, site-directed mutagenesis and affinity labelling of the cyclodipeptide synthase AlbC, we demonstrate that the covalent intermediate reacts with the aminoacyl moiety of the second aminoacyl tRNA, forming a dipeptidyl enzyme, and identify the aminoacyl-binding sites of the aminoacyl tRNAs.

\footnotetext{
${ }^{1}$ Service d'Ingénierie Moléculaire des Protéines, iBiTec-S, Commissariat à l'Energie Atomique et aux Energies Alternatives (CEA), 91191 Gif-sur-Yvette, France. ${ }^{2}$ Laboratoire de Biochimie, Unité mixte de Recherche 7654, Ecole Polytechnique, Centre National de la Recherche Scientifique, 91120 Palaiseau, France.

* These authors contributed equally to this work. Correspondence and requests for materials should be addressed to M.G. (email: muriel.gondry@cea.fr) or to Y.M. (email: yves.mechulam@polytechnique.edu).
} 
C yclodipeptide synthases (CDPSs) are a novel family of enzymes that hijack aminoacyl tRNAs (aa-tRNAs) from their canonical function in ribosomal protein synthesis to produce various cyclodipeptides, which are precursors of many secondary metabolites with diverse biological activities ${ }^{1-3}$. They employ a ping-pong-type catalytic mechanism beginning with the binding of a first aa-tRNA and the transfer of its aminoacyl moiety onto a conserved serine leading to the formation of an aminoacyl enzyme intermediate ${ }^{4-7}$. The subsequent steps of the catalytic mechanism have not yet been elucidated. It has been proposed that the aminoacyl enzyme reacts with the aminoacyl moiety of a second aa-tRNA to form either a dipeptidyl enzyme ${ }^{6}$ or a dipeptidyl tRNA intermediate ${ }^{5}$, which undergoes intramolecular cyclization to generate the final cyclodipeptide product (Fig. 1). Neither of these two putative intermediates has ever been detected directly.

The crystal structures of three CDPSs have been determined: AlbC from Streptomyces noursei, Rv2275 from Mycobacterium tuberculosis and YvmC from Bacillus licheniformis ${ }^{4-6}$. These enzymes possess a common architecture very similar to the catalytic domains of class-I aa-tRNA synthetases (aaRSs), especially class-Ic TyrRSs and TrpRSs. In particular, the aminoacyl moiety of the first aa-tRNA substrate of CDPS is thought to bind in a pocket ${ }^{4-7}$, structurally corresponding to the tyrosine-binding pocket in TyrRSs $\left(\mathrm{PDB} 1 \mathrm{~J}^{8} \mathrm{U}^{8}\right)$. Accordingly, buffer components were identified within this pocket in several CDPS crystal structures ${ }^{4,6}$. However, no structure of a CDPS in complex with either a natural substrate or a ligand representative of a reaction intermediate has been obtained.

Here we present the first crystal structure of a CDPS complexed with a reaction intermediate analogue. This structure, combined with site-directed mutagenesis and chemical biology experiments, reveals that the second reaction intermediate is a dipeptidyl enzyme. We map the aminoacyl-binding sites for each of the two aa-tRNA substrates. Finally, we identify the residue responsible for the intramolecular cyclization of the dipeptidyl intermediate and revisit the roles of the other active site residues.

\section{Results}

We reasoned that the identification of the second reaction intermediate would be facilitated by blocking the enzymatic reaction before dipeptide cyclization. To identify residues possibly involved in cyclization, we first determined the crystal structure of a complex mimicking a putative dipeptidyl AlbC intermediate.
AlbC synthesizes cyclo(L-Phe-L-Leu) (cFL) and cyclo(L-Phe-L-Phe) $(\mathrm{cFF})^{2}$, and its $\mathrm{S} 37$ residue is the target for phenylalanylation ${ }^{4}$ (Fig. 1). Therefore, if a dipeptidyl enzyme intermediate indeed occurs, it should be composed of a dipeptide covalently linked by its carboxylate terminus to the $\mathrm{S} 37$ hydroxyl. To obtain a structural analogue of L-Phe-L-Phe-AlbC, we used N-carbobenzyloxy-L-Phechloromethyl ketone (ZPCK) (Fig. 2a), a molecule close to the PhePhe dipeptide (Fig. 1). We used the active AlbC variant $S 37 \mathrm{C}^{4}$ as a target for ZPCK labelling, because the chloromethyl ketone in this analogue is able to react with thiol groups (Fig. 2a). As expected, trypsin digestion and peptide mass fingerprinting (PMF) showed that C37 was covalently modified after incubation of AlbC S37C with ZPCK (Supplementary Fig. 1).

We solved the structure of the modified AlbC S37C, named AlbC S37C-ZPK (ZPK corresponds to N-carbobenzyloxy-L-Phemethyl ketone), by molecular replacement using the previously determined unmodified AlbC structure (PDB 3OQV). The resulting electron density map showed a supplementary mass of electron density connected to $\mathrm{C} 37$, and some rearrangements in the structure of the enzyme. The supplementary density clearly corresponded to ZPK (Supplementary Fig. 2). The structure was refined at $2.9 \AA$ resolution after a few cycles of model fitting and energy minimization (Table 1). The structural model consists of the entire AlbC S37C enzyme bound to ZPK (Fig. 2b), excluding residues 1-9 and residues 234-239 at the C-terminal extremity for which no electron density was visible. Overall, the AlbC S37CZPK structure is highly similar to the unmodified AlbC structure (root mean squared deviation $=0.9 \AA$ over $169 \mathrm{C} \alpha$, Supplementary Figs 3 and 4). However, the region linking the $\beta 6$ and $\beta 7$ strands, in the vicinity of the ZPCK binding site, is rearranged (residues 202-225). The 202-207 loop and $\alpha 8$ (residues 208-213) are pushed away from the active site cavity in the AlbC S37C-ZPK structure (Fig. 2b and Supplementary Figs 3 and 4). In the structure of unmodified AlbC (PDB 3OQV), Y202 and neighbouring residues are involved in crystal packing (Supplementary Fig. 5). Therefore, the conformational difference of the 202-225 region between AlbC S37C-ZPK and unmodified AlbC cannot be attributed to modification by ZPK. Notably, the conformation of this region in AlbC S37C-ZPK appears close to that observed in Rv2275 and YvmC (PDB 2X9Q and 3OQJ, respectively; Supplementary Fig. 4).

The aromatic ring (Phe1) of ZPK is buried deep within a hydrophobic pocket, corresponding to that occupied by cyclodithiothreitol in the AlbC structure ${ }^{4}$, or by the buffer components CHES or CAPSO in the structure of $\mathrm{YvmC}^{6}$. The cavity is

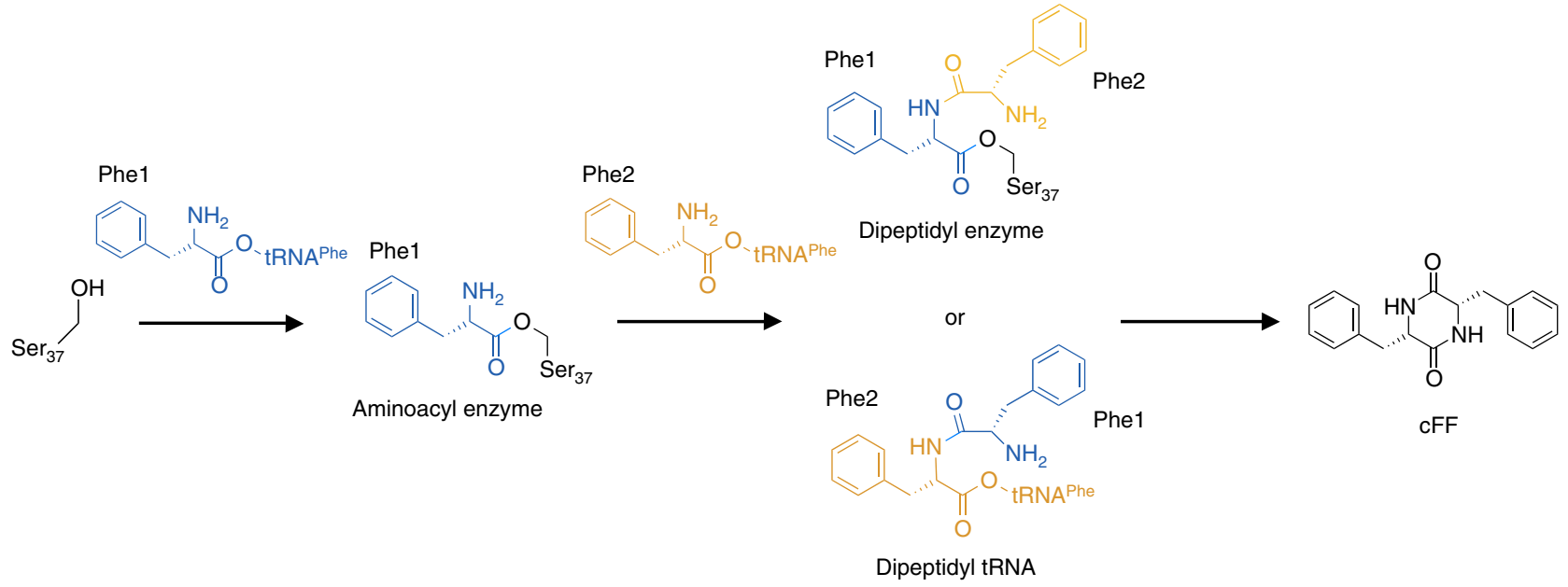

Figure 1 | Formation of cyclo(L-Phe-L-Phe) catalysed by the CDPS AlbC. The existence of the phenylalanyl AlbC intermediate was demonstrated previously ${ }^{4}$, but the nature of the dipeptidyl intermediate was unknown. 
a

AlbC S37C

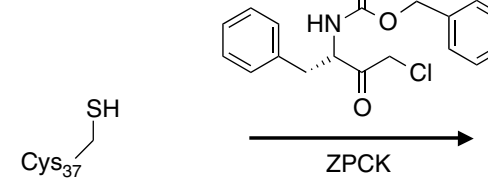

AlbC S37C-ZPK<smiles>O=C(N[C@@H](Cc1ccccc1)C(=O)CSCC([Mg])[Ge])OCc1ccccc1</smiles>

b

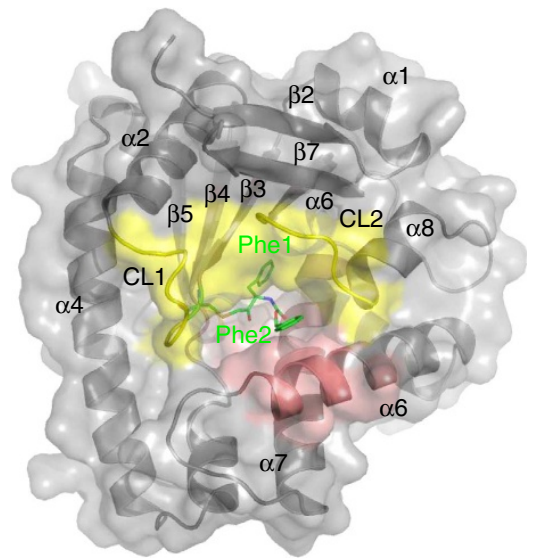

C

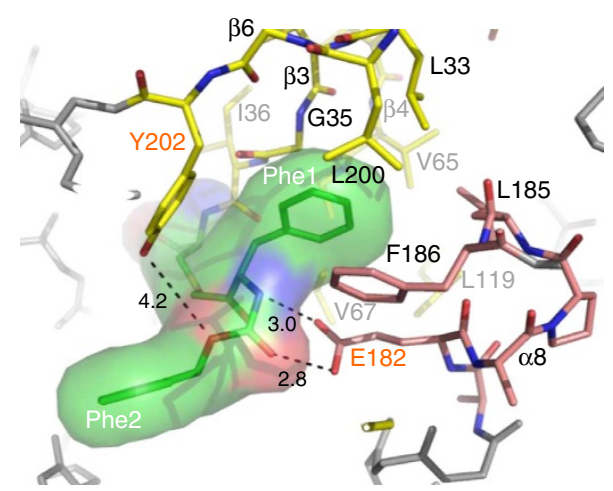

d

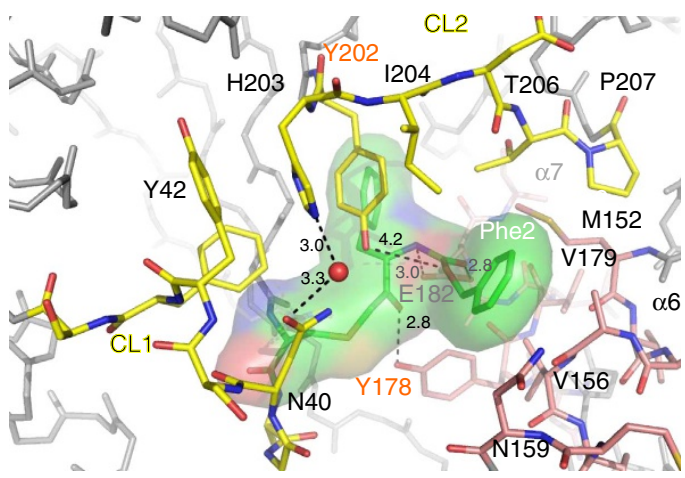

e

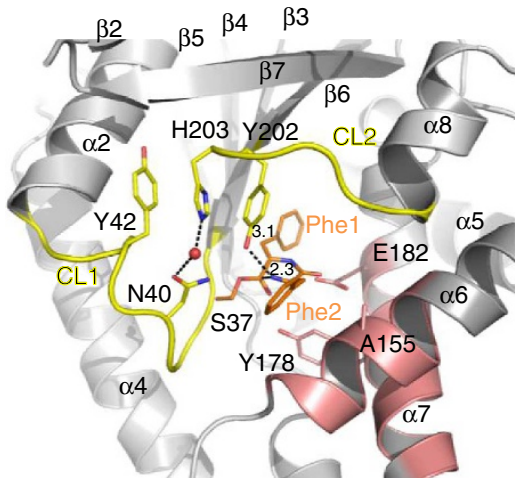

Figure 2 | Affinity labelling of AlbC and crystal structure of a complex mimicking the dipeptidyl enzyme intermediate. (a) The AlbC variant S37C was covalently modified with $\mathrm{N}$-carbobenzyloxy-L-Phe-chloromethyl ketone (ZPCK). The labelled CDPS was named AlbC S37C-ZPK. (b) Cartoon representation of the structure of AlbC S37C-ZPK (PDB 4Q24). Secondary structure elements are labelled. The protein is shown in grey as a semitransparent molecular surface. Regions surrounding the ZPK adduct are coloured as follows: CL1 and CL2 (CL1: 33-43, CL2: 202-207), and the N terminus of $\beta 3,4,6$ are in yellow. Parts of $\alpha 6$ (151-159) and $\alpha 7$ (172-182) are in pink. Phe1 is buried deep within its cavity, whereas Phe2 lies in a wide pocket. (c) Close-up view of the Phe1-binding site. The ZPK adduct is shown as green sticks within its molecular surface. Residues delineating the cavities are shown as sticks; catalytic residues are labelled in orange. Yellow is used for $\beta$-strands and pink for helix $\alpha 8$. Relevant interactions are indicated with a dashed line. (d) Close-up view of the Phe2-binding site. Yellow is used for CL1 and CL2, and pink for $\alpha 6$ and $\alpha 7$. Important residues are shown as sticks; catalytic residues are labelled in orange. This view highlights stacking interactions between Y42, H2O3 and Y202, and a water-mediated interaction between N40 and H2O3 that stabilize the positions of CL1 and CL2. Notably, a water molecule is present at the same position in the active site of Rv2275. Y178, E182 and Y202 are in close proximity to ZPK. Hydrogen bonds between Y178 and E182 are indicated with dashed lines. (e) Model of the diphenylalanyl enzyme. Position of the diphenylalanyl adduct was modelled according to the position of the ZPK adduct (Supplementary Fig. 6). The diphenylalanyl adduct was constructed with the REEL and eLBOW programmes from the Phenix suite ${ }^{28}$. Important regions involved in the binding of the dipeptide are in yellow and pink. The dipeptide intermediate is shown as orange sticks. Important residues are shown as sticks. According to this model, the oxygen group of Y202 is positioned 3.1 $\AA$ away from the $\mathrm{NH}_{2}$ group of the dipeptide. All structural drawings were done with PYMOL (www.pymol.org).

delineated on one side by strands $\beta 3, \beta 4$ and $\beta 6$, and on the other side by helix $\alpha 8$. In particular, there is a stacking interaction between F186 and the Phe1 ring (Fig. 2c). In the S37C-ZPK structure, the benzyl ring (Phe2) lies in a wider cavity delineated by two loops, hereafter named CL1 and CL2 (catalytic loop 1: residues 36-43; catalytic loop 2: residues 202-207), the C-terminal part of $\alpha 6$ (152-159) and the central part of $\alpha 7$ (175-183) (Fig. 2d). Stacking interactions involving Y42, H203 and Y202, and a water-mediated interaction between N40 and H203, stabilize the positions of the two catalytic loops. Notably, in
AlbC S37C-ZPK the position of CL1 and CL2 places the side chain of Y202 in proximity to ZPK (Fig. 2c,d).

We derived a model of the dipeptidyl enzyme from the AlbC S37C-ZPK structure (Fig. 2e and Supplementary Fig. 6) and used it to identify residues possibly involved in dipeptide cyclization. The intramolecular cyclization of the dipeptide ester requires that the dipeptide be in a cis conformation to place the primary ammonium group in close proximity to the ester, ${ }^{9}$. The conformation adopted by the dipeptide places the ammonium group of Phe 2 near the ester carbonyl ( $2.3 \AA)$, which is in line 


\section{Table 1 | Data collection and refinement statistics for AlbC S37C-ZPK structure determination.}

Native $^{\star}$

\begin{tabular}{lc}
\hline Data collection & \\
Space group & $\mathrm{P}_{2} 21$ \\
Cell dimensions & $86.97,86.97,70.74$ \\
$a, b, c(\AA)$ & $90.0,90.0,120.0$ \\
$\alpha, \beta, \gamma\left({ }^{\circ}\right)$ & $37.66,2.9$ \\
Resolution $(\AA)$ & $0.078(0.618)^{\dagger}$ \\
$R_{\text {sym }}$ & $11.45(1.75)$ \\
$l / \sigma l$ & $98.1 \%(98.5)$ \\
Completeness (\%) & $2.71(2.72)$ \\
Redundancy & \\
& \\
Refinement & $37.66,2.9$ \\
Resolution $(\AA)$ & 7001 \\
No. reflections & $0.2007 / 0.2526$ \\
$R_{\text {work }} / R_{\text {free }}$ & 1,773 \\
No. atoms & 28 \\
Protein & 3 \\
Modified residue & Protein 71 \\
Waters & Modified residue 75 \\
$B$-factors $\left(\AA^{2}\right)$ & Waters 54 \\
& \\
Root mean squared deviations & 0.009 \\
Bond lengths $(\AA)$ & 1.07 \\
Bond angles $\left({ }^{\circ}\right)$ & \\
\hline
\end{tabular}

${ }^{\star}$ A single crystal was used for data collection.

†Values in brackets correspond to the highest-resolution shell.

with this requirement (Fig. 2e and Supplementary Fig. 6). This primary ammonium group should be deprotonated by a catalytic base to generate the nucleophile for attack at the activated ester. The conserved Y202 residue is a good candidate to fulfill this role, because its hydroxyl would be close to the ammonium group of Phe2 (3.1 A) (Fig. 2e and Supplementary Fig. 6). Such a role for a $\mathrm{Y}$ residue is unusual but has already been described ${ }^{1-13}$. In Rv2275 and YvmC, this residue was initially proposed to position and increase the nucleophilicity of the catalytic serine ${ }^{5,6}$. However, we showed previously that its substitution with a phenylalanine in AlbC did not affect the formation of the aminoacyl enzyme intermediate, demonstrating that Y202 is not responsible for the activation of the catalytic serine ${ }^{4}$. The Y202 hydroxyl is nevertheless essential, because the Y202F variant has 20 -fold lower enzymatic activity than the wild-type enzyme, although its structural integrity is not affected (Supplementary Table 1 and Supplementary Fig. 7). We hypothesized that the modification of Y202 to F may impair the cyclization process, allowing the trapping of the dipeptidyl enzyme if it indeed occurs. We incubated briefly the Y202F variant with two equivalents of Phe-tRNA ${ }^{\text {Phe }}$ and immediately analysed the resulting species by liquid chromatography-mass spectrometry (LC-MS) or PMF. LC-MS analysis showed a mixture of three species, with masses consistent with the unmodified AlbC Y202F, a phenylalanyl enzyme and a diphenylalanyl enzyme (Supplementary Fig. 8). Previous PMF analyses of trypsin-digested AlbC and of its Y202F variant gave two fragments containing the catalytic S37 (fragments [29-46] and [27-46]) ${ }^{4}$ (Supplementary Fig. 9). The PMF analysis of Y202F incubated with two equivalents of PhetRNA $^{\text {Phe }}$ gave the [27-46] fragment, low amounts of the [29-46] fragment and two additional fragments (Fig. 3). The $\mathrm{m} / z$ of these two fragments were 2,310.17 and 2,457.2, and corresponded to the values expected for the addition of phenylalanyl and diphenylalanyl moieties on fragment [27-46], respectively. We sequenced unambiguously by both $b$ - and $y$-series the fragment with an $\mathrm{m} / \mathrm{z}$ of 2,457.2, which showed that it contained a diphenylalanyl adduct on S37. This experiment thus demonstrates the formation of the covalent dipeptidyl enzyme intermediate. Moreover, we did not observe the diphenylalanyl adduct with wild-type AlbC; therefore, we conclude that Y202 is involved in the cyclization process, probably by deprotonating the primary ammonium group.

The AlbC S37C-ZPK structure allowed us to revisit the role of other active site residues and propose the following catalytic mechanism (Fig. 4). When the first Phe-tRNA ${ }^{\text {Phe }}$ binds, the phenylalanyl side chain is accommodated in the catalytic pocket and both amino and carbonyl groups are appropriately positioned through hydrogen bonds with the side chains of E182 and Y178 (Step 1). The Y178 hydroxyl polarizes the incoming ester carbonyl of the first aa-tRNA and facilitates the nucleophilic attack by the S37 hydroxyl. S37 activation probably results from a concerted proton shuffling mechanism involving the two adjacent vicinal hydroxyls of the nucleotide A76 of the tRNA moiety ${ }^{4}$, as proposed for the ribosome at the peptidyl transferase centre ${ }^{14}$ and the FemX aa-transferase of Weissella viridescens ${ }^{15}$. Then, the Y178 hydroxyl may help to stabilize the resulting phenylalanyl enzyme intermediate (Step 2). This is consistent with our structural data, indicating that Y178 is engaged in a polar contact with the carbonyl of Phe1 (Fig. 2d), and our previous finding showing that the Y178F substitution substantially impairs both enzymatic activity and the formation of the phenylalanyl enzyme intermediate ${ }^{4}$. Such a role for a Y hydroxyl has been described previously for prolyl oligopeptidase ${ }^{16}$ and cocaine

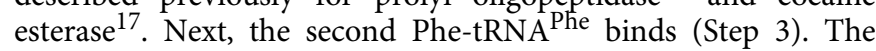
formation of the subsequent diphenylalanyl enzyme intermediate implies that the ammonium group of the phenylalanyl enzyme intermediate has to be deprotonated by a catalytic base to perform the nucleophilic attack on the incoming carbonyl of the second substrate. The nearby E182 may fulfill this role ${ }^{13,18,19}$. This residue is essential to the catalytic reaction and is involved in catalytic steps following amino-acyl formation ${ }^{4-6}$. Thus, the ammonium group of the first phenylalanine, deprotonated by the nearby carboxyl of E182, may attack the carbonyl group of the second Phe-tRNA ${ }^{\text {Phe }}$ to generate a diphenylalanyl enzyme intermediate (Step 4). The carboxyl of E182 now in its protonated state may form electrostatic contacts with the two carbonyls of the dipeptide, similar to those we observed in the AlbC S37CZPK structure (Fig. 2c). The successive protonation states of E182 are supported further by $\mathrm{pKa}$ predictions made with the heuristic pKa calculation software PROPKA ${ }^{20}$ (pKa values of 7.8 in free AlbC, 4.8 in the aminoacyl enzyme and 7.5 in AlbC S37C-ZPK). Thus, E182 and Y178 form hydrogen bonds with the dipeptide, precisely holding its backbone in the conformation required for cyclization. Y202 is then properly positioned to deprotonate the ammonium group of the second amino acid. Y202 participates in a hydrogen bond network, also involving N40 and H203, which are essential for the accurate positioning of catalytic residues. The substitution of either residue with alanine resulted in a dramatic impairment of enzymatic activity (Supplementary Table 1) and phenylalanyl enzyme formation (Supplementary Fig. 10), whereas the structural integrity of the variants was not affected (Supplementary Fig. 7). Finally, Y202 would deprotonate the primary ammonium group to generate the nucleophile for attack at the enzyme ester bond (Step 5), leading to the formation of the cyclodipeptide product.

The AlbC-ZPK structure enables the identification of the two pockets $\mathrm{P} 1$ and $\mathrm{P} 2$ accommodating the aminoacyl moieties of the first and second aa-tRNA, respectively (Fig. 2c,d). We compared the residues lining $\mathrm{P} 1$ and $\mathrm{P} 2$ in all characterized CDPSs together with the cyclodipeptides that these enzymes 

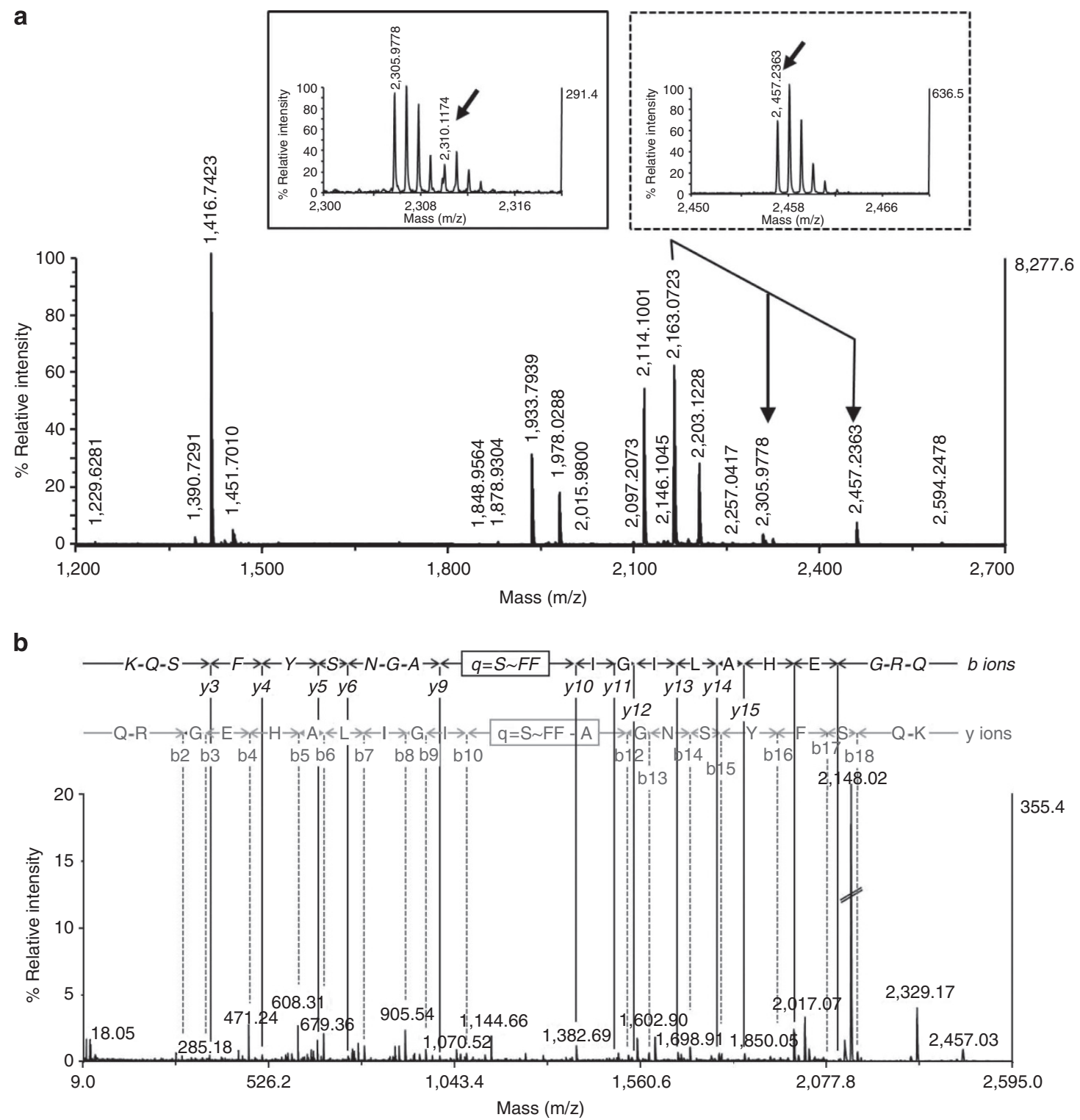

Figure 3 | Identification of the covalent dipeptidyl enzyme intermediate with the variant Y202F. (a) PMF analysis of trypsin-digested Y202F variant preincubated with two equivalents of Phe-tRNA ${ }^{\text {Phe }}$ for $30 \mathrm{~s}$. Arrows indicate the formation of two additional fragments that were not observed in the PMF analysis of the trypsin-digested Y202F variant not incubated with Phe-tRNA Phe (Supplementary Fig. 9): the $\mathrm{m} / \mathrm{z}$ at 2,310.12 (close-up view in solid box) corresponds to the potential phenylalanylated fragment [27-46] and the $m / z$ at 2,457.24 (close-up view in dashed box) corresponds to the diphenylalanylated fragment [27-46]. The non-modified [27-46] fragment was detected at $m / z 2,163.07$. (b) PSD-MS/MS spectrum of $m / z$ 2,457.24. Both $b$ - and $y$-ion series identify the amino acid sequence of the modified AlbC fragment [27-46] and identify the residue 37 as the diphenylalanylated residue. The main daughter-ion at $m / z$ 2,148.02 corresponds to the modified fragment [27-46] without its diphenylalanyl moiety. Note that the scale of the 2,148.02 peak differs from the others.

produce (Supplementary Table 2 and Supplementary Fig. 11). P1 has a hydrophobic character, in keeping with the accommodation of hydrophobic amino acids. Our structural data show that P2 is wider than P1, consistent with a somewhat relaxed specificity for the second aminoacyl moiety. In line with this idea, the amino acids lining P2 are poorly conserved (Supplementary Table 2).

\section{Discussion}

This study, combining structural approaches and chemical biology experiments, elucidates the missing step in the mechanism of the recently discovered CDPSs-the formation of a dipeptidyl enzyme.
Our work also identifies two different binding sites for the aminoacyl moieties of the two aa-tRNA substrates. We previously identified two different regions accommodating the tRNA moieties of the substrates ${ }^{21}$. Taken together, these data provide insight into the molecular basis of the interaction between CDPSs and their substrates. The first aa-tRNA binds to the enzyme through the accommodation of its aminoacyl moiety in the P1 pocket and the interaction of its tRNA moiety with basic residues located in the $\alpha 4$ helix that form a basic patch at the surface of the protein. When the acyl enzyme is formed, the second aa-tRNA binds with its aminoacyl moiety accommodated in the $\mathrm{P} 2$ pocket and its tRNA moiety interacting with residues of the $\alpha 6-\alpha 7$ loop. In both TyrRS and TrpRS, the loop equivalent to this $\alpha 6-\alpha 7$ loop is involved in tRNA binding $8,22-24$. CDPSs may have retained 


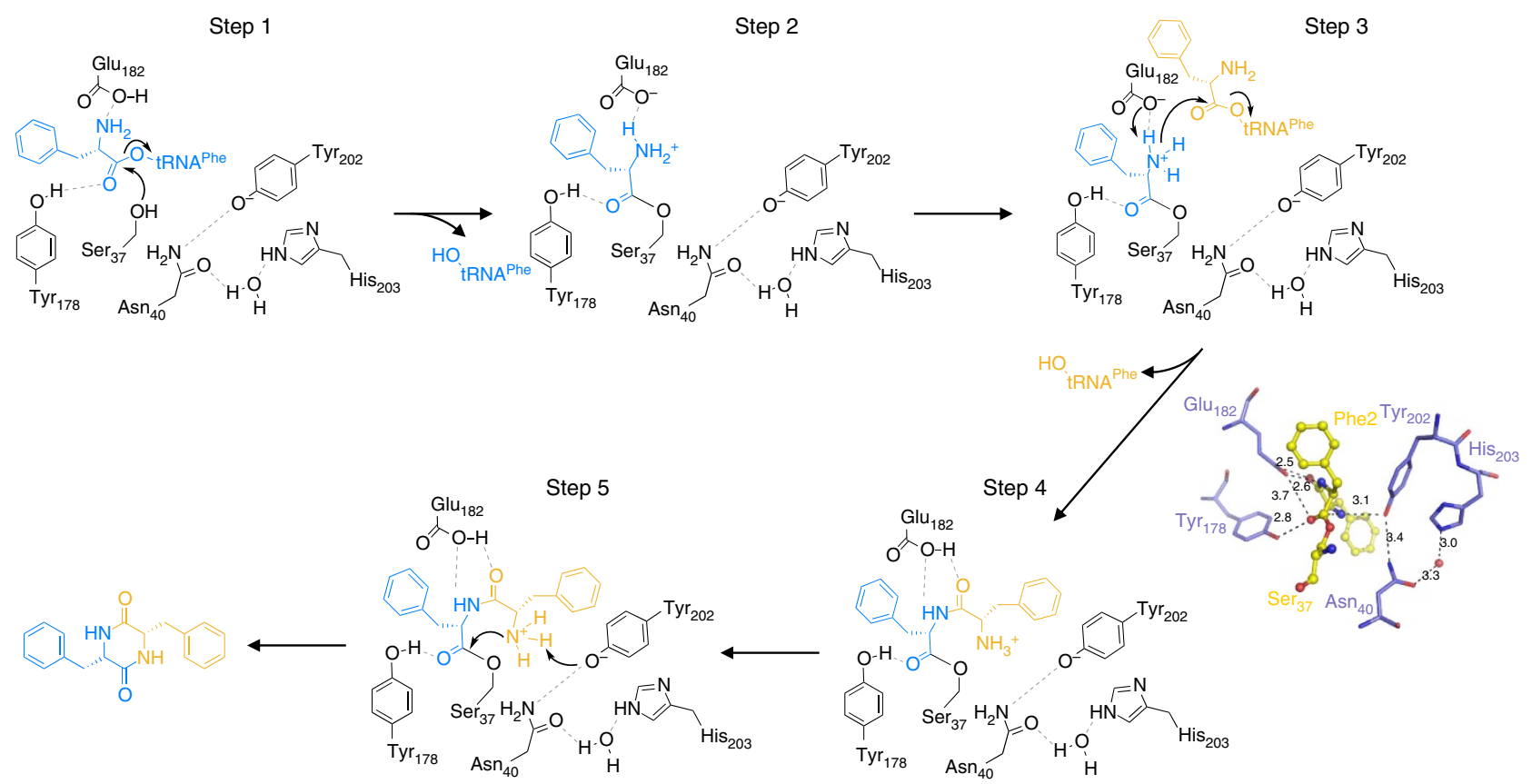

Figure 4 | Proposed mechanism for cyclodipeptide synthesis by CDPSs during which a covalent acyl- and subsequently dipeptidyl-AlbC are formed. The formation of cFF catalysed by AlbC is shown; the first and second incorporated phenylalanyl moieties of Phe-tRNA ${ }^{\text {Phe }}$ are highlighted in blue and yellow, respectively. Possible interactions are indicated by dashed lines and predicted distances are labelled. Step 4 is illustrated by the model of the diphenylalanyl enzyme intermediate in which residues involved in the catalysis are shown as blue sticks (see Supplementary Fig. 6). The amino groups of Phe-tRNAs (Steps 1 and 3 ) are assumed to be in their $\mathrm{NH}_{2}$ state, as described in the context of the ribosome ${ }^{32}$. The amino groups of the aminoacyl- (Steps 2 and 3) and dipeptidyl intermediates (Steps 4 and 5) are expected to be in their $\mathrm{NH}_{3}^{+}$state ${ }^{33}$; these protonation states are supported by pKa values predicted with the heuristic pKa calculation software PROPKA ${ }^{20}$ (10.4 and 11.2, respectively).

some characteristics of class-Ic aaRSs regarding their interaction with the tRNA moiety of the second substrate. Further studies describing precisely the tRNA-binding regions on CDPSs are still needed to clarify this issue.

This study indicates that compared with class-Ic aaRSs, CDPSs have acquired both new active site residues and a new aminoacylbinding site for a second aa-tRNA substrate. This is an important step forward in the understanding of CDPS specificity, and this knowledge may facilitate the engineering of these enzymes to produce a diversity of natural and unnatural cyclodipeptides.

\section{Methods}

Purification of AlbC variants. Plasmids encoding the AlbC variants S37C, Y202F and N40A were constructed previously ${ }^{4}$. The variant $\mathrm{H} 203 \mathrm{~A}$ was obtained via PCR mutagenesis with oligonucleotides described in Supplementary Table 3 of the plasmid pQE60-AlbC encoding C-terminal $\mathrm{His}_{6}$-tagged AlbC according to the QuikChange site-directed mutagenesis method (Stratagene). Sequences were verified by DNA sequencing. The $\mathrm{C}$-terminal $\mathrm{His}_{6}$-tagged proteins were produced and purified as described previously ${ }^{4}$. Purified proteins were quantified by ultraviolet spectrophotometry. Protein molecular weights were verified by electrospray ionization MS (Esquire HCT ion trap mass spectrometer (Bruker Daltonik, GmbH)). The structural integrity of the variants was verified by far-ultraviolet circular dichroism spectroscopy (Jasco J-815 circular dichroism spectropolarimeter).

Crystallization and structure determination. A mixture of AlbC S37C $(75 \mu \mathrm{M})$ and ZPCK $(100 \mu \mathrm{M})$ was used for crystallization trials. Crystals were obtained by the sitting drop method at $4{ }^{\circ} \mathrm{C}$ (Mosquito robot, TTP Labtech), by mixing equal volumes of protein and a solution containing $10 \%$ (W/v) PEG8000, $50 \mathrm{mM} \mathrm{KCl}$, $50 \mathrm{mM}$ sodium cacodylate, $\mathrm{pH} 6.0$ (condition E8 of the Natrix screen, Hampton Research). Cryoprotection was achieved by adding $25 \%(\mathrm{v} / \mathrm{v})$ glycerol to the crystallization liquor. Diffraction data were collected at $100 \mathrm{~K}(\lambda=0.984 \AA)$ on the Proxima-1 beamline at the SOLEIL synchrotron equipped with a Pilatus detector. Diffraction images were analysed with X-ray Detector Software (XDS) ${ }^{25}$ and the data were processed with programmes of the CCP4 package ${ }^{26}$. The final data set was at a resolution of $2.9 \AA$ (Table 1). The structure was solved by molecular replacement with PHASER ${ }^{27}$ and the structure of $\mathrm{AlbC}^{4}$ was used as a search model (PDB 3OQV). Density for ZPCK connected to C37 was easily identified. The geometry restraints file for the ZPCK-modified cysteinyl residue was created with eLBOW and REEL ${ }^{28}$. The structure was refined through several cycles of manual rebuilding with $\mathrm{COOT}^{29}$ and positional refinement with BUSTER (BUSTER version 2.10.0., Global Phasing Ltd, Cambridge, UK). A final round of refinement was performed with single group translation/libration/screw (TLS) parameters. The final model (free $R=0.253, R=0.201$ ) contained all AlbC residues, including the ZPCK-modified C37, with the exception of residues 1-9 at the amino terminus and residues $234-239$ at the $C$ terminus, as well as three water molecules. Refinement statistics are given in Table 1. A model of the dipeptidyl enzyme was generated to facilitate interpretation by building a diphenylalanyl-modified serine residue from the refined ZPCK-modified cysteine coordinates and optimizing the geometry with eLBOW $^{28}$.

Acyl tRNA synthesis. Production and purification of Escherichia coli tRNAs were performed as described previously ${ }^{30}$. tRNA $^{\text {Phe }}$ was expressed from pBSTNAV2/ tRNA $^{\text {Phe }}$ (ref. 31) and tRNA ${ }^{\text {LeuCAG }}$ from pBSTNAV2/tRNA ${ }^{\text {LeuCAG }}$ (ref. 21). The concentration of aminoacylated tRNA was determined from plateau charging experiments ${ }^{21}$.

Identification of the covalent peptidyl-enzyme species. A total of $12 \mu \mathrm{M}$ ( 2 equivalents) of Phe-tRNA ${ }^{\text {Phe }}$ was mixed with $6 \mu \mathrm{M}$ of AlbC (or variant) in $20 \mu \mathrm{l}$ of the reaction buffer containing $50 \mathrm{mM}$ HEPES-KOH pH 7.0 and $50 \mathrm{mM} \mathrm{KCl}$. After $30 \mathrm{~s}$ of incubation, a $5-\mu \mathrm{l}$ aliquot was removed and submitted to hydrolysis for further analysis by PMF: the samples were diluted up to $10 \mu \mathrm{l}$ in $50 \mathrm{mM}$ $\mathrm{NH}_{4} \mathrm{AcO}_{2}, \mathrm{pH} 8.0$, and $1 \mu \mathrm{l}$ of porcine trypsin (Promega) $\left(400 \mathrm{ng}^{-1} \mathrm{l}^{-1}\right.$ ) was added. Trypsinolysis was performed at $50^{\circ} \mathrm{C}$ up to $10 \mathrm{~min}$. Sample digests were then acidified by $1.25 \%$ trifluoroacetic acid (TFA) in water, desalted and concentrated by C18 ziptip and spotted with 4-HCCA matrix solution $\left(10 \mathrm{mg} \mathrm{ml}^{-1}\right.$ in $49.8 / 49.8 /$ 0.4: $\mathrm{CH}_{3} \mathrm{CN} / \mathrm{H}_{2} \mathrm{O} / \mathrm{TFA}: \mathrm{v} / \mathrm{v} / \mathrm{v}$ ) on a matrix-assisted laser desorption/ionization plate. The remaining reaction sample was analysed by LC-MS (ESI-ion trap Esquire HCT Bruker Daltonics) with a linear gradient of acetonitrile/water with $0.1 \%$ TFA (ProSwift RP-4H monolithic column from Thermo-Scientific).

MS and MS/MS spectra were registered with a 4800 MALDI-TOF/TOF mass spectrometer (Applied Biosystems, Foster City, USA) in positive reflectron mode Each MS spectrum was the result of 1,000 laser shots and trypsin autolysis fragments were used as internal standards for calibration.

MS/MS analyses were performed under PSD (post-source decay) mode. Each MS/MS spectrum was the result of 10,000 shots. The sequence of the trypsin fragments was identified with Data Explorer processing software (Version 4.9, 
Applied Biosystems) to assign the potential chemical modifications of the serine or cysteine residues.

AlbC S37C-ZPK characterization. AlbC S37C $\left(0.04 \mu \mathrm{g} \mu \mathrm{l}^{-1}\right)$ was incubated with 100 equivalents of ZPCK in Tris- $\mathrm{HCl} 100 \mathrm{mM}$, $\mathrm{pH} 8$ for $3 \mathrm{~h}$. The sample was loaded onto a Ni-NTA agarose resin (Qiagen) to wash out the excess ZPCK. The resin was washed with a large excess of $50 \mathrm{mM}$ HEPES-KCl, $50 \mathrm{mM} \mathrm{KCl}$ buffer. A 5- $\mu \mathrm{l}$ aliquot of resin was removed, diluted up to $10 \mu \mathrm{l}$ in $50 \mathrm{mM} \mathrm{NH}_{4} \mathrm{AcO}_{2}, \mathrm{pH}$ 8.0, and submitted to trypsin digestion for PMF as described for the identification of the peptidyl enzyme intermediate.

Cyclodipeptide-synthesizing activity assay. The CDPS activity of AlbC and $\mathrm{AlbC}$ variants was measured with an end point assay, as performed previously ${ }^{21}$. Activity was determined in a coupled assay containing aaRSs to generate in situ the aa-tRNA substrates. The assay was performed in a buffer containing $50 \mathrm{mM}$ HEPES-KOH, pH 7.5, $50 \mathrm{mM} \mathrm{KCl,} 15 \mathrm{mM} \mathrm{MgCl}$, $0.1 \mathrm{mM}$ EDTA, $2 \mathrm{mM}$ ATP, $10 \mathrm{mM} \beta$-mercaptoethanol), with $50 \mu \mathrm{M}$ Phe and Leu, $1 \mu \mathrm{M}$ PheRS and LeuRS, $150 \mathrm{nM}$ AlbC or $1.5 \mu \mathrm{M}$ AlbC variant, $0.75 \mu \mathrm{M}$ Phe-tRNA ${ }^{\text {Phe }}$ and $0.66 \mu \mathrm{M}$ LeutRNA ${ }^{\mathrm{LeuCAG}}$. The reaction was carried out at $30^{\circ} \mathrm{C}$ with a preincubation of $15 \mathrm{~min}$ with the aaRSs for the synthesis of aa-tRNAs before the addition of AlbC or AlbC variant. The enzymatic reaction was initiated by the addition of AlbC or AlbC variant. The reaction was stopped after 21 min by acidification with $2 \%$ TFA, and mixed with known concentrations of stable isotope internal standards $\left({ }^{13} \mathrm{C}_{9}{ }^{15} \mathrm{~N}\right.$ labelled cFF and cFL solutions), before cFF and cFL quantification by LC-MS as described previously ${ }^{7}$.

\section{References}

1. Lautru, S., Gondry, M., Genet, R. \& Pernodet, J. L. The albonoursin gene cluster of S. noursei: biosynthesis of diketopiperazine metabolites independent of nonribosomal peptide synthetases. Chem. Biol. 9, 1355-1364 (2002).

2. Gondry, M. et al. Cyclodipeptide synthases are a family of tRNA-dependent peptide bond-forming enzymes. Nat. Chem. Biol. 5, 414-420 (2009).

3. Belin, P. et al. The nonribosomal synthesis of diketopiperazines in tRNAdependent cyclodipeptide synthase pathways. Nat. Prod. Rep. 29, 961-979 (2012).

4. Sauguet, L. et al. Cyclodipeptide synthases, a family of class-I aminoacyl-tRNA synthetase-like enzymes involved in non-ribosomal peptide synthesis. Nucleic Acids Res. 39, 4475-4489 (2011).

5. Vetting, M. W., Hegde, S. S. \& Blanchard, J. S. The structure and mechanism of the Mycobacterium tuberculosis cyclodityrosine synthetase. Nat. Chem. Biol. 6, 797-799 (2010).

6. Bonnefond, L. et al. Structural basis for nonribosomal peptide synthesis by an aminoacyl-tRNA synthetase paralog. Proc. Natl Acad. Sci. USA 108, 3912-3917 (2011).

7. Seguin, J. et al. Nonribosomal peptide synthesis in animals: the cyclodipeptide synthase of Nematostella. Chem. Biol. 18, 1362-1368 (2011).

8. Kobayashi, T. et al. Structural basis for orthogonal tRNA specificities of tyrosyl-tRNA synthetases for genetic code expansion. Nat. Struct. Biol. 10, 425-432 (2003).

9. Purdie, J. E. \& Benoiton, N. L. Piperazinedione formation from esters of dipeptides containing glycine, alanine, and sarcosine - kinetics in aqueoussolution. J. Chem. Soc. Perk. Transact. 2, 1845-1852 (1973).

10. Steinberg, S. M. \& Bada, J. L. Peptide decomposition in the neutral $\mathrm{Ph}$-region via the formation of diketopiperazines. J. Org. Chem. 48, 2295-2298 (1983).

11. Agarwal, V., Pierce, E., McIntosh, J., Schmidt, E. W. \& Nair, S. K. Structures of cyanobactin maturation enzymes define a family of transamidating proteases. Chem. Biol. 19, 1411-1422 (2012).

12. Frey, P. A. \& Hegeman, A. D. Chemical and stereochemical actions of UDP-galactose 4-epimerase. Accounts Chem. Res. 46, 1417-1426 (2013).

13. Schlippe, Y. V. G. \& Hedstrom, L. A twisted base? The role of arginine in enzyme-catalyzed proton abstractions. Arch. Biochem. Biophys. 433, 266-278 (2005).

14. Rodnina, M. V. The ribosome as a versatile catalyst: reactions at the peptidyl transferase center. Curr. Opin. Struct. Biol. 23, 595-602 (2013).

15. Fonvielle, M. et al. Decoding the logic of the tRNA regiospecificity of nonribosomal FemX(Wv) aminoacyl transferase. Angew. Chem. Int. Ed. Engl. 49, 5115-5119 (2010).

16. Fulop, V., Bocskei, Z. \& Polgar, L. Prolyl oligopeptidase: an unusual betapropeller domain regulates proteolysis. Cell 94, 161-170 (1998).

17. Turner, J. M. et al. Biochemical characterization and structural analysis of a highly proficient cocaine esterase. Biochemistry 41, 12297-12307 (2002).

18. Harris, T. K. \& Turner, G. J. Structural basis of perturbed pKa values of catalytic groups in enzyme active sites. IUBMB Life 53, 85-98 (2002).
19. Grimsley, G. R., Scholtz, J. M. \& Pace, C. N. A summary of the measured pK values of the ionizable groups in folded proteins. Protein Sci. 18, 247-251 (2009).

20. Sondergaard, C. R., Olsson, M. H. M., Rostkowski, M. \& Jensen, J. H. Improved treatment of ligands and coupling effects in empirical calculation and rationalization of pK(a) values. J. Chem. Theory Comput. 7, 2284-2295 (2011).

21. Moutiez, M. et al. Specificity determinants for the two tRNA substrates of the cyclodipeptide synthase AlbC from Streptomyces noursei. Nucleic Acids Res. 42, 7247-7258 (2014).

22. Jia, J. et al. Two essential regions for tRNA recognition in Bacillus subtilis tryptophanyl-tRNA synthetase. Biochem. J. 365, 749-756 (2002).

23. Jia, J. et al. Residues Lys-149 and Glu-153 switch the aminoacylation of tRNA(Trp) in Bacillus subtilis. J. Biol. Chem. 279, 41960-41965 (2004).

24. Yaremchuk, A., Kriklivyi, I., Tukalo, M. \& Cusack, S. Class I tyrosyl-tRNA synthetase has a class II mode of cognate tRNA recognition. EMBO J. 21, 3829-3840 (2002).

25. Kabsch, W. Evaluation of single-crystal X-ray-diffraction data from a position-sensitive detector. J. Appl. Crystallogr. 21, 916-924 (1988).

26. Collaborative Computational Project, Number 4. The Ccp4 suite - programs for protein crystallography. Acta. Crystallogr. D. Biol. Crystallogr. 50, 760-763 (1994).

27. Storoni, L. C., McCoy, A. J. \& Read, R. J. Likelihood-enhanced fast rotation functions. Acta. Crystallogr. D. Biol. Crystallogr. 60, 432-438 (2004).

28. Moriarty, N. W., Grosse-Kunstleve, R. W. \& Adams, P. D. Electronic Ligand Builder and Optimization Workbench (eLBOW): a tool for ligand coordinate and restraint generation. Acta. Crystallogr. D. Biol. Crystallogr. 65, 1074-1080 (2009).

29. Emsley, P., Lohkamp, B., Scott, W. G. \& Cowtan, K. Features and development of Coot. Acta. Crystallogr. D. Biol. Crystallogr. 66, 486-501 (2010).

30. Mechulam, Y., Guillon, L., Yatime, L., Blanquet, S. \& Schmitt, E. Protection-based assays to measure aminoacyl-tRNA binding to translation initiation factors. Methods Enzymol. 430, 265-281 (2007).

31. Meinnel, T., Mechulam, Y. \& Fayat, G. Fast purification of a functional elongator tRNAMet expressed from a synthetic gene in vivo. Nucleic Acids Res. 16, 8095-8096 (1988).

32. Johansson, M. et al. $\mathrm{pH}$-sensitivity of the ribosomal peptidyl transfer reaction dependent on the identity of the A-site aminoacyl-tRNA. Proc. Natl Acad. Sci. USA 108, 79-84 (2011).

33. Barta, A., Dorner, S. \& Polacek, N. Mechanism of ribosomal peptide bond formation. Science 291, 203 (2001).

\section{Acknowledgements}

We thank Vincent Dive for helpful discussion and Jean-Baptiste Charbonnier for advice about the initial crystallogenesis assays. This work was supported by the Commissariat à l'Energie Atomique et aux Energies Alternatives (CEA) and the French National Research Agency (ANR 2010/Blan 1501 01). The Service d'Ingénierie Moléculaire des Protéines is member of the Laboratory of Excellence LERMIT.

\section{Author contributions}

Funding was obtained by M.G. Experiments were conceived and designed by M.M. and M.G. J.S. and E.F. purified AlbC and prepared aminoacylated tRNA Phe . M.M. and P.B. cloned and purified the AlbC variants. M.M. and R.T. performed the MS analyses. J.S. carried out circular dichroism experiments. J.S. and E.S. performed the crystallogenesis assays, and E.S. and Y.M. determined the crystal structure of AlbC S37C-ZPK. The manuscript was drafted by M.M., E.S., Y.M. and M.G., and was revised by all authors.

\section{Additional information}

Accession codes: The atomic coordinates and the structure factors have been deposited with the accession code 4Q24.

Supplementary Information accompanies this paper at http://www.nature.com/ naturecommunications

Competing financial interests: The authors declare no competing financial interests.

Reprints and permission information is available online at http://www.nature.com/ reprintsandpermissions/

How to cite this article: Moutiez, M. et al. Unravelling the mechanism of non-ribosomal peptide synthesis by cyclodipeptide synthases. Nat. Commun. 5:5141 doi: 10.1038/ ncomms6141 (2014). 